\title{
The order parameter in binary mixtures
}

\author{
O.V.Patsahan \\ Institute for Condensed Matter Physics \\ of the National Academy of Sciences of Ukraine, \\ 1 Svientsitskii Str., 290011 Lviv, Ukraine
}

Received August 31, 1998, in final form November 30, 1998

\begin{abstract}
Phase transitions in a binary mixture are investigated by means of the collective variables method with a reference system. It is shown that the system is described with two sets of collective variables: $\eta_{\vec{k}}$ and $\xi_{\vec{k}}$. The CV connected with the order parameter is $\eta_{\vec{k}=0}$ in the case of the gas-liquid critical point as well as in the case of the mixing-demixing phase transition. The particular form of $\eta_{0}$ for each of these phenomena can be determined by means of the relations between the microscopic parameters, temperature, density and concentration of the system under consideration.
\end{abstract}

Key words: binary mixture, collective variables, phase transition, order parameter

PACS: 05.70.Fh, 05.70.Jk, 64.10.+h

\section{Introduction}

The choice of the order parameter in multicomponent fluid mixtures is a serious problem because the character of the phase transition can change continuously from the pure gas-liquid transition to a mixing-demixing one. The question of the physical nature of the order parameter in binary fluid mixtures has been considered until recently from the point of view of both the phenomenological theory $[1,2]$ and the microscopic approach [3]-[6]. Nowadays the commonly accepted idea is that both the gas-liquid and mixing-demixing phase transitions are accompanied by total density fluctuations as well as by relative density fluctuations. The only symmetrical mixture exhibits a complete distinction between these two processes [6]. However, such an "ideal" system is not likely to occur in reality. In real mixtures the contribution from each type of the fluctuation processes changes along the critical curve. The evaluation of such contributions at each critical curve point is essential to the definition of the order parameter and to the understanding of the phase transition character in the mixture. In our approach the question of the physical nature of the order parameter seems to have a consistent and clear solution. 
In this paper we propose a microscopic approach to the study of phase transitions in multicomponent fluids. It is based on the method of collective variables (CV) with a distinguished reference system (RS) $[7,8]$. The point is that the statistical description of the phase transition is to be performed in the appropriate phase space specific to a certain physical model. Among the independent variables of this space there should be those connected with order parameters. This phase space forms a set of CV . Each of them is a mode of density fluctuations corresponding to the specific feature of the model under consideration. In particular, for a magnetic system the CV are variables connected with spin density fluctuation modes, for a one-component fluid - with particle density fluctuation modes. What is the content of the CV for a multicomponent system? We will answer this question below.

\section{Functional representation of the grand partition function of a binary mixture}

Let us consider a classical two-component system of interacting particles consisting of $N_{a}$ particles of species $a$ and $N_{b}$ particles of species $b$. The volume of the system is $V$, the system temperature is $T$.

Let us assume that the interaction in the system has a pairwise additive character. The interaction potential between a $\gamma$ particle at $\vec{r}_{i}$ and a $\delta$ particle at $\vec{r}_{j}$ may be expressed as a sum of two terms:

$$
U_{\gamma \delta}\left(r_{i j}\right)=\psi_{\gamma \delta}\left(r_{i j}\right)+\phi_{\gamma \delta}\left(r_{i j}\right)
$$

where $\psi_{\gamma \delta}(r)$ is a potential of a short-range repulsion that will be chosen as an interaction between two hard spheres $\sigma_{\gamma \gamma}$ and $\sigma_{\delta \delta} . \phi_{\gamma \delta}(r)$ is an attractive part of the potential which dominates at large distances. An arbitrary positive function belonging to the $L_{2}$ class can be chosen as the potential $\phi_{\gamma \delta}(r)$.

Further consideration of the problem is done in the extended phase space: in the phase space of the Cartesian coordinates of the particles and in the CV phase space. An interaction connected with repulsion (potential $\psi_{\gamma \delta}(r)$ ) is considered in the space of the Cartesian coordinates of the particles. We call this two-component hard spheres system a reference system (RS). The interaction connected with an attraction (potential $\phi_{\gamma \delta}(r)$ ) is considered in the CV space. The phase space overflow is cancelled by introducing the transition Jacobian. The contribution of the shortrange forces to the long-range interaction screening is ensured by averaging this Jacobian over the RS.

Then a grand partition function in the CV representation with a RS can be written as

$$
\Xi=\Xi_{0} \Xi_{1}
$$

where $\Xi_{0}$ is the grand partition function of the RS. The thermodynamic and structural properties of the RS are assumed to be known. We assume that in the region of temperatures, concentrations and densities we are interested in, the thermodynamic 
functions of the RS remain analytic. $\Xi_{1}$ has the following form:

$$
\begin{gathered}
\Xi_{1}=\int(\mathrm{d} \rho)(\mathrm{d} c) \exp \left[\beta \mu_{1}^{+} \rho_{0}+\beta \mu_{1}^{-} c_{0}-\frac{\beta}{2 V} \sum_{\vec{k}}\left[\tilde{V}(k) \rho_{\vec{k}} \rho_{-\vec{k}}\right.\right. \\
\left.\left.+\tilde{W}(k) c_{\vec{k}} c_{-\vec{k}}+\tilde{U}(k) \rho_{\vec{k}} c_{-\vec{k}}\right]\right] J(\rho, c) .
\end{gathered}
$$

Here the following notations are introduced:

$\rho_{\vec{k}}$ and $c_{\vec{k}}$ are the CV connected with total density fluctuation modes and relative density (or concentration) fluctuation modes in the binary system.

Functions $\mu_{1}^{+}$and $\mu_{1}^{-}$have the form:

$$
\mu_{1}^{+}=\frac{\sqrt{2}}{2}\left(\mu_{1}^{a}+\mu_{1}^{b}\right), \quad \mu_{1}^{-}=\frac{\sqrt{2}}{2}\left(\mu_{1}^{a}-\mu_{1}^{b}\right)
$$

and are determined from the equations

$$
\begin{gathered}
\frac{\partial \ln \Xi_{1}}{\partial \beta \mu_{1}^{+}}=\langle N\rangle, \\
\frac{\partial \ln \Xi_{1}}{\partial \beta \mu_{1}^{-}}=\left\langle N_{a}\right\rangle-\left\langle N_{b}\right\rangle ; \\
\tilde{V}(k)=\left(\tilde{\phi}_{a a}(k)+\tilde{\phi}_{b b}(k)+2 \tilde{\phi}_{a b}(k)\right) / 2, \\
\tilde{W}(k)=\left(\tilde{\phi}_{a a}(k)+\tilde{\phi}_{b b}(k)-2 \tilde{\phi}_{a b}(k)\right) / 2, \\
\tilde{U}(k)=\left(\tilde{\phi}_{a a}(k)-\tilde{\phi}_{b b}(k)\right) / 2, \\
J(\rho, c)=\int(\mathrm{d} \omega)(\mathrm{d} \gamma) \exp \left[\mathrm{i} 2 \pi \sum_{v e c k}\left(\omega_{k} \rho_{k}+\gamma_{k} c_{k}\right)\right] J(\omega, \gamma), \\
J(\omega, \gamma)=\exp \left[\sum_{n \geqslant 1} \sum_{i_{n} \geqslant 0} \frac{(-\mathrm{i} 2 \pi)^{n}}{n !} \sum_{\vec{k}_{1} \ldots \vec{k}_{n}} M_{n}^{\left(i_{n}\right)}(0, \ldots, 0)\right. \\
\left.\quad \times \gamma_{\vec{k}_{1}} \ldots \gamma_{\vec{k}_{i_{n}}} \omega_{\vec{k}_{i_{n+1}}} \ldots \omega_{\vec{k}_{n}}\right] .
\end{gathered}
$$

Index $i_{n}$ is used to indicate the number of variables $\gamma_{\vec{k}}$ in the cumulant expansion (2.6). Cumulants $M_{n}^{\left(i_{n}\right)}$ are expressed as linear combination of the partial cumulants $M_{\gamma_{1} \ldots \gamma_{n}}$ and are presented for $\gamma_{1}, \ldots, \gamma_{n}=a, b$ and $n \leqslant 4$ in [6] (see Appendix B in $[6])$.

Formulas (2.1)-(2.7) are the initial formulas in our study of phase transitions in binary fluids. 


\section{The order parameter in a binary mixture}

Let us consider the functional integral in Gaussian approximation (2.1)-(2.7). This approximation, also known as the random-phase approximation, yields the correct qualitative picture of the phenomena under consideration. After integration over variables $\gamma_{k}$ and $\omega_{k}, \Xi_{1}$ can be rewritten as

$$
\begin{aligned}
\Xi_{1}^{G}=\frac{1}{2 \pi} & \prod_{\vec{k}}^{\prime} \frac{1}{\pi} \frac{1}{\sqrt{\Delta(k)}} \int(\mathrm{d} \rho)(\mathrm{d} c) \exp \left[\rho_{0}\left(\beta \mu_{1}^{+}+\aleph_{1} / \Delta\right)\right. \\
& +c_{0}\left(\beta \mu_{1}^{-}+\aleph_{2} / \Delta\right)-\left(M_{1}^{(0)} \aleph_{1}+M_{1}^{(1)} \aleph_{2}\right) \\
& \left.-\frac{1}{2} \sum_{\vec{k}}\left[\rho_{\vec{k}} \rho_{-\vec{k}} A_{11}(k)+c_{\vec{k}} c_{-\vec{k}} A_{22}(k)+2 \rho_{\vec{k}} c_{-\vec{k}} A_{12}(k)\right]\right]
\end{aligned}
$$

where

$$
\begin{aligned}
\aleph_{1}=M_{2}^{(2)} M_{1}^{(0)}-M_{2}^{(1)} & M_{1}^{(1)}, \quad \aleph_{2}=M_{2}^{(0)} M_{1}^{(0)}-M_{2}^{(1)} M_{1}^{(0)}, \\
A_{11}(k) & =-\frac{1}{2}\left(\frac{\beta}{V} \tilde{V}(k)+\frac{M_{2}^{(2)}}{\Delta}\right), \\
A_{22}(k) & =-\frac{1}{2}\left(\frac{\beta}{V} \tilde{W}(k)+\frac{M_{2}^{(0)}}{\Delta}\right), \\
A_{12}(k) & =-\frac{1}{2}\left(\frac{\beta}{V} \tilde{U}(k)-\frac{M_{2}^{(1)}}{\Delta}\right), \\
\Delta & =M_{2}^{(0)} M_{2}^{(2)}-\left(M_{2}^{(1)}\right)^{2} .
\end{aligned}
$$

In order to determine the phase space of the $\mathrm{CV}$ connected with the order parameters we introduce independent collective excitations by diagonalizing the square form in (3.1) by means of the orthogonal transformation:

$$
\begin{aligned}
\rho_{\vec{k}} & =A(k) \eta_{\vec{k}}+B(k) \xi_{\vec{k}}, \\
c_{\vec{k}} & =C(k) \eta_{\vec{k}}+D(k) \xi_{\vec{k}} .
\end{aligned}
$$

The explicit forms for coefficients $A(k), B(k), C(k)$ and $D(k)$ are given in Appendix A.

As a result, we have

$$
\begin{aligned}
\Xi_{1}^{G}= & \frac{1}{2 \pi} \prod_{\vec{k}}^{\prime} \frac{1}{\pi} \frac{1}{\sqrt{\Delta(k)}} \int(\mathrm{d} \eta)(\mathrm{d} \xi) \exp \left[\eta_{0}\left(A M_{1}+C M_{2}\right)+\xi_{0}\left(B M_{1}+D M_{2}\right)\right. \\
& \left.-\left(M_{1}^{(0)} \aleph_{1}+M_{1}^{(1)} \aleph_{2}\right) / \Delta(0)-\frac{1}{2} \sum_{\vec{k}}\left(\varepsilon_{11}(k) \eta_{\vec{k}} \eta_{-\vec{k}}+\varepsilon_{22}(k) \xi_{\vec{k}} \xi_{-\vec{k}}\right)\right],
\end{aligned}
$$

where

$$
\varepsilon_{i i}(k)=-\left(A_{11}(k)+A_{22}(k) \mp \sqrt{\left(A_{11}(k)-A_{22}(k)\right)^{2}+4 A_{12}^{2}(k)}\right) .
$$


One of the quantities (3.5) (or both) tends to zero as the critical temperature is approached at a certain wave vector $\vec{k}^{*}$. Thus the CV $\eta_{\vec{k}^{*}}\left(\right.$ or $\xi_{\vec{k}^{*}}$ ) can be identified as the order parameter, where the wave vector $\vec{k}^{*}$ should correspond to the minimum of one of the functions $\varepsilon_{11}(k)$ or $\varepsilon_{22}(k)$ (or both). These functions depend on temperature, the attractive potentials $\tilde{\phi}_{\gamma \delta}(k)$ and the characteristics of the RS. The RS enters into (3.5) via the cumulants $M_{\gamma \delta}(k) . M_{\gamma \delta}(k)$ can be expressed by the Fourier transforms of the direct correlation functions $C_{\gamma \delta}(k)$ using the OrnsteinZernike equations for a mixture.

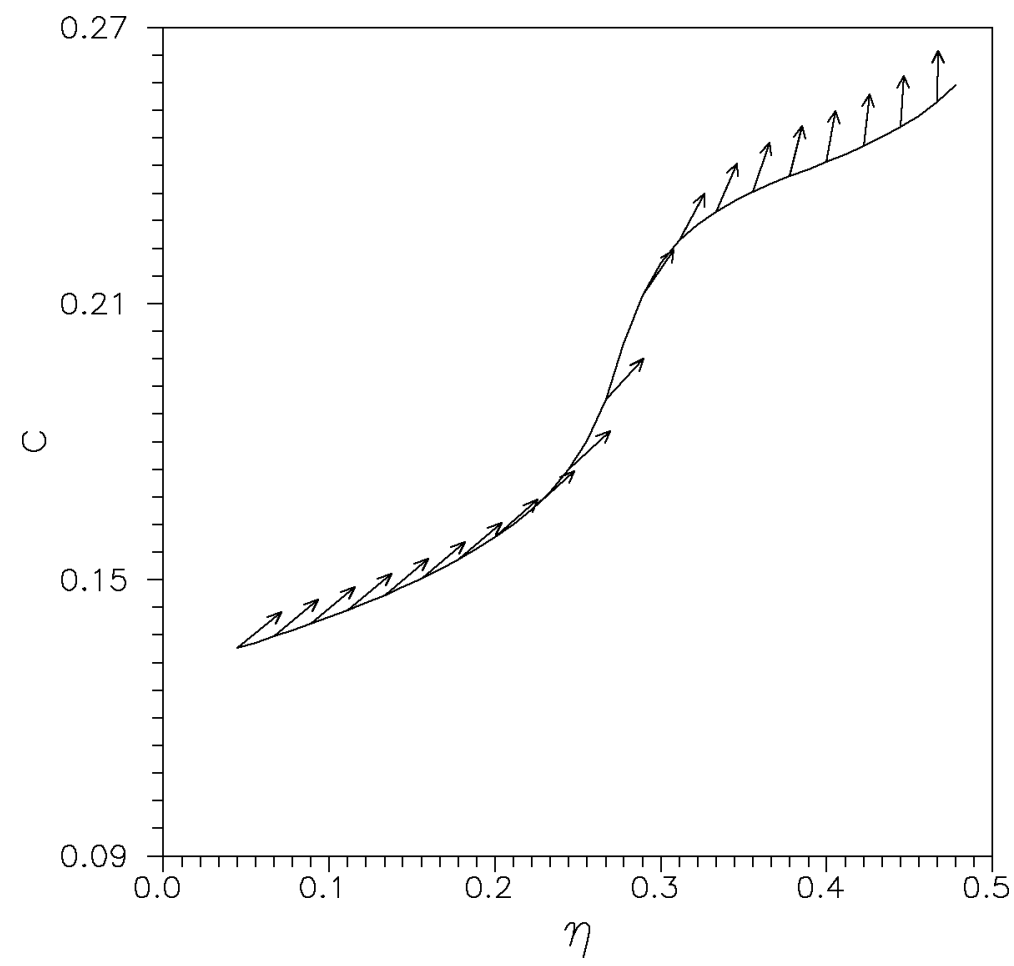

Figure 1. Density-concentration projection of the mean field critical line of the model binary mixture at $\alpha=1.0, q=0.9$ and $r=0.6\left(\alpha=\sigma_{a a} / \sigma_{b b}, q=\right.$ $\left.-\tilde{\phi}_{b b}(0) /\left|\tilde{\phi}_{a a}(0)\right|, r=-\tilde{\phi}_{b b}(0) /\left|\tilde{\phi}_{a b}(0)\right|\right)$.

Coefficients $\varepsilon_{11}(k)$ and $\varepsilon_{22}(k)$ are studied both as wave vector functions at different values of temperature $T$, density and concentration including the gas-liquid and mixing-demixing critical points [9] and as temperature functions at $\vec{k}=0$ [10]. The results show that branch $\varepsilon_{11}(k)$ is the first to reach zero no matter whether the system approaches the gas-liquid or gas-gas demixing critical point. Moreover, $\varepsilon_{11}(k)$ and $\varepsilon_{22}(k)$ have the minima at $\vec{k}=0[9]$. Hence we can draw the following conclusions:

1. Branch $\varepsilon_{11}(k)$ is always critical.

2. Because $\varepsilon_{11}(k)$ has the minimum at $\vec{k}=0$, the $\mathrm{CV}$ connected with the order parameter is $\eta_{0}$ in the case of the gas-liquid critical point as well as in the case of the mixing-demixing phase transition. The particular form of $\eta_{0}$ for each 
of these phenomena can be determined by means of the relations between the microscopic parameters, temperature, density and concentration of the system,

e.g. by means of coefficients $A(0), B(0), C(0)$ and $D(0)$.

Thus, the proposed approach enables us, on microscopic grounds, to define the order parameter at each point along a critical curve and to understand the character of the phase transition in the binary mixture. Figure 1 shows the $(\eta, c)$ projection of the $(T, \eta, c)$ critical surface of the model binary mixture ( $\eta$ is the packing density of the mixture, $c$ is the concentration of species $b$ ). The arrows show the direction of the strong fluctuations (order parameter) along the critical curve.

Based on the Gaussian distribution (3.1)-(3.2) we have determined the critical branch and, correspondingly, the $\mathrm{CV} \eta_{0}$ connected with the order parameter. The purpose of our further study will be calculating the binary mixture behaviour in the vicinity of its critical points.

\section{Appendix A}

The coefficients $A(k), B(k), C(k)$ and $D(k)$ have the forms:

$$
\begin{aligned}
A= & \sqrt{2}\left|A_{12}\right|\left[4 A_{12}^{2}+\left(A_{11}-A_{22}\right)^{2}-\left(A_{11}-A_{22}\right) \sqrt{\left(A_{11}-A_{22}\right)^{2}+4 A_{12}^{2}}\right]^{-1} \\
B= & \sqrt{2}\left|A_{12}\right|\left[4 A_{12}^{2}+\left(A_{11}-A_{22}\right)^{2}+\left(A_{11}-A_{22}\right) \sqrt{\left(A_{11}-A_{22}\right)^{2}+4 A_{12}^{2}}\right]^{-1} \\
C= & -\frac{\sqrt{2}}{2} \frac{\left|A_{12}\right|}{\left(A_{12}\right)}\left[A_{11}-A_{22}-\sqrt{\left(A_{11}-A_{22}\right)^{2}+4 A_{12}^{2}}\right] \\
& \times\left[4 A_{12}^{2}+\left(A_{11}-A_{22}\right)^{2}-\left(A_{11}-A_{22}\right) \sqrt{\left(A_{11}-A_{22}\right)^{2}+4 A_{12}^{2}}\right]^{-1} \\
D= & -\frac{\sqrt{2}}{2} \frac{\left|A_{12}\right|}{\left(A_{12}\right)}\left[A_{11}-A_{22}+\sqrt{\left(A_{11}-A_{22}\right)^{2}+4 A_{12}^{2}}\right] \\
& \times\left[4 A_{12}^{2}+\left(A_{11}-A_{22}\right)^{2}+\left(A_{11}-A_{22}\right) \sqrt{\left(A_{11}-A_{22}\right)^{2}+4 A_{12}^{2}}\right]^{-1} .
\end{aligned}
$$

\section{References}

1. Anisimov M.A., Gorodetskii E.E., Kulikov V.D., Sengers J.V. // Phys. Rev. E, 1995, vol. 51, No. 2, p. 1199-1215.

2. Anisimov M.A., Gorodetskii E.E., Kulikov V.D., Povodyrev A.A., Sengers J.V. // Physica A, 1995, vol. 220, p. 277-324.

3. Chen X.S., Forstmann F. // J. Chem. Phys., 1992, vol. 97, No. 5, p. 3696-3703.

4. Parola A., Reatto L. // J. Phys.: Condens. Matter, 1993, vol. 5, p. B165-B172.

5. Parola A., Reatto L. // Advan. in Phys. 1995, vol. 44, No. 3, p. 211-298.

6. Yukhnovskii I.R., Patsahan O.V. // J. Stat. Phys., 1995, vol. 81, No. 3/4, p. 647-672.

7. Yukhnovskii I.R., Holovko M.F. Statistical Theory of Classical Equilibrium Systems. Naukova Dumka, Kiev, 1980.

8. Patsagan O.V., Yukhnovskii I.R. // Theor. Math. Phys., 1990, vol. 83, No. 1, p. 387397. 
9. Patsahan O.V., The phase transitions in binary systems. I. Random phase approximation. Preprint Inst. Cond. Matter Phys. Acad. Sci. Ukraine, ICMP-92-2U, Lviv, 1992 (in Ukrainian).

10. Patsahan O.V. // Ukr. Fiz. Zh., 1996, vol. 41, No. 9, p. 877-884 (in Ukrainian).

\section{Параметр порядку у бінарних сумішах}

\section{О.В.Пацаган}

Інститут фізики конденсованих систем НАН України, 290011 Львів, вул. Свєнціцького, 1

Отримано 31 серпня 1998 р., в остаточному вигляді 30 листопада 1998 р.

Фазові переходи у бінарній суміші досліджуються з допомогою методу колективних змінних з виділеною системою відліку. Показано, що система описується двома наборами колективних змінних: $\eta_{\vec{k}} \mathbf{i}$ $\xi_{\vec{k}}$. Колективною змінною,зв'язаною з параметром порядку є змінна $\eta_{\vec{k}=0}$ як у випадку критичної точки газ-рідина, так і у випадку фазового переходу змішування-незмішування. Конкретна форма $\eta_{0}$ для кожного з цих явищ визначається співвідношенням між температурою, густиною, концентрацією і мікроскопічними параметрами системи, яка розглядається.

Ключові слова: бінарна суміш, колективна змінна, фазовий перехід, параметр порядку

PACS: 05.70.Fh, 05.70.Jk, 64.10. $+h$ 
